\title{
Integrative analysis of DNA methylation, mRNAs, and small RNAs during maize embryo dedifferentiation
}

\author{
Hongjun Liu ${ }^{1,2 \dagger}$, Langlang Ma ${ }^{1 \dagger}$, Xuerong Yang ${ }^{2 \dagger}$, Lin Zhang ${ }^{3}$, Xing Zeng ${ }^{3}$, Shupeng Xie ${ }^{4}$, Huanwei Peng ${ }^{5}$, \\ Shibin Gao ${ }^{1}$, Haijian Lin', Guangtang Pan ${ }^{1}$, Yongrui Wu ${ }^{6}$ and Yaou Shen ${ }^{1 *}$
}

\begin{abstract}
Background: Maize (Zea mays) is an important model crop for transgenic studies. However, genetic transformation of maize requires embryonic calli derived from immature embryo, and the impact of utilizing tissue culture methods on the maize epigenome is poorly understood. Here, we generated whole-genome MeDIP-seq data examining DNA methylation in dedifferentiated and normal immature maize embryos.

Results: We observed that most of the dedifferentiated embryos exhibited a methylation increase compared to normal embryos. Increased methylation at promoters was associated with down-regulated protein-coding gene expression; however, the correlation was not strong. Analysis of the callus and immature embryos indicated that the methylation increase was induced during induction of embryonic callus, suggesting phenotypic consequences may be caused by perturbations in genomic DNA methylation levels. The correlation between the 21-24nt small RNAs and DNA methylation regions were investigated but only a statistically significant correlation for 24nt small RNAs was observed.
\end{abstract}

Conclusions: These data extend the significance of epigenetic changes during maize embryo callus formation, and the methylation changes might explain some of the observed embryonic callus variation in callus formation.

Keywords: Embryo callus, Epigenome, Maize, MeDIP_seq, 24 nt small RNAs

\section{Background}

Maize is one of the most important crops for both human and livestock animals. For several decades, maize has been modified using both conventional and molecular breeding methods to generate plants with an increased yield and a greater ability to adapt to various disadvantageous conditions. Efforts are also underway to create maize plants with improved yield traits and resistance to various stresses using genetic engineering techniques.

Genetically modified maize plants are usually generated via tissue culture, and maize has been a primary target for genetic manipulation. To date, genetic transformation of maize still largely depends on immature maize embryoderived calli [1]. Genetically, maize is a diverse species $[2,3]$

\footnotetext{
* Correspondence: shenyaou@gmail.com

${ }^{\dagger}$ Equal contributors

${ }^{1}$ Key Laboratory of Biology and Genetic Improvement of Maize in Southwest Region, Maize Research Institute, Sichuan Agricultural University, Chengdu 611130, China

Full list of author information is available at the end of the article
}

with a complex genome encoding repetitive regions $[4,5]$. However, methylation changes occur and are an important source of tissue culture-induced variation, which appears to be much more frequent than genetic sequence variation [6] and suggests that epigenetic mechanisms play a critical role in the cellular transformation and, ultimately, cellular phenotypes. There is evidence that epigenetic alternations in both plants and animals can lead to phenotypic variations [7-11]. However, the role of epigenetic variation, in particular during maize embryo callus induction, has not been well characterized.

Generally, plant genomic DNA is methylated in three cytosine contexts: CG, $\mathrm{CHG}$, and $\mathrm{CHH}(\mathrm{H}=\mathrm{A}, \mathrm{T}$, or $\mathrm{C})$. Previous studies have indicated that distinct genetic pathways participate in distinct methytransferase-regulated DNA methylation in these contexts in Arabidopsis [12]. However, the majority of genome-wide methylation studies were performed in Arabidopsis and in different maize lines and tissues [13-21]. In these studies, DNA methylation was 
closely associated with transposable elements and repetitive DNA. In general, methylation of promoter regions is correlated with gene expression, whereas methylation changes to gene body regions show low/no correlation with gene expression [14, 17]. More interestingly, little evidence reports consistent changes to maize DNA methylation patterns in response to specific and distinct stress treatments [21]. To some extent, the maize embryo callus can be induced under certain stress-like conditions such as induction by auxin/cytokinin or wounding, and although the induction conditions are different from specific stress treatments, we suspect that DNA methylation patterns change during maize embryo callus formation.

Therefore, we investigated the effect of callus initiation through dedifferentiation on the methylome of maize embryos. We generated genome-wide DNA methylation maps using methylated DNA immunoprecipitation sequencing (MeDIP-seq) in dedifferentiated maize embryos after callus induction and in normal immature maize embryos without induction. We observed that tissue culture of the embryos induced changes to DNA methylation. In most cases, we observed an increase in DNA methylation throughout the genome that was associated with small RNA expression (specially 24 nt small RNA), and these methylation changes were enriched at promoter regions. Elevated DNA methylation at promoters in dedifferentiated embryos was associated with alterations in the expression levels of particular genes.

\section{Methods}

\section{Plant materials}

Maize inbred line18-599R, a cultivar with high dedifferentiation capacity, was used in this study. It was cultivated and is currently kept by Maize Research Institute of Sichuan Agricultural University. For DNA preparation in this study, all inbred line 18-599R seedlings, previously described in [22], were grown in the growth chamber at $27{ }^{\circ} \mathrm{C}$ with humidity of $70 \%$. In brief, after 12 days (d) of self-pollination, immature ear of each plant was harvested. The immature embryos $(1.5 \mathrm{~mm}-1.8 \mathrm{~mm})$ were isolated and cultured with optimized N6 medium aseptically at $27^{\circ} \mathrm{C}$ in darkness for $15 \mathrm{~d}$. Generally, after inoculating, the immature embryos were divided into three stages according to their morphological features [22]: 1-5 d (intumescent embryo, Stage I), 6-10 d (initial callus formation, Stage II), and 11-15 d (embryonic callus formation, Stage III). Samples were collected from three individuals each day and pooled for three biological replicates at each stage. The embryos from immediately harvested ears without inoculation were collected with three biological replicates and used as a control group $(0 \mathrm{~d}, \mathrm{CK})$ in this study.

\section{DNA extraction and methylated DNA}

\section{immunoprecipitation sequencing (MeDIP-seq)}

Genomic DNA was extracted from the samples using TaKaRa Universal Genomic DNA Extraction Kit Ver. 3.0
(DV811A) (TaKaRa, Osaka, Japan) according to the manufacturer's instructions. In total, 12 genomic DNA samples (three biological replicates at each of the four stages) were sonicated to produce DNA fragments ranging from $100 \mathrm{bp}$ to $500 \mathrm{bp}$. After DNA end-repair and 3'dA-tailing using the Paired-end DNA Sample Prep Kit (Illumina, San Diego, CA, USA), the DNA samples were ligated to Illumina sequencing primer adaptors. Double-stranded DNA was denatured and immunoprecipitated using an anti-5methycytosine monoclonal antibody (Zymo Research, Orange, CA, USA). For each sample, the following procedures were performed as described [23]. $220 \mathrm{bp}$ to $320 \mathrm{bp}$ bands were excised and purified from the immunoprecipitation gel and quantified using an Agilent 2100 Analyzer (Agilent Technologies, Santa Clara, CA, USA). Finally, ultra-high-throughput 50 bp paired-end sequencing was performed using the Illumina HiSeq 2000 (BGI, Shenzhen, China) according to the manufacturer's protocols.

Paired-ended sequencing raw reads (PE $50 \mathrm{bp)}$ generated from MeDIP-seq were used to remove the containing adaptors and low quality reads with default settings. The clean reads (remaining reads) were aligned to the maize genome (RefGen_v3) [5] using Soap2 [24], allowing up to 2 bp mismatches to the reference genome and only returning uniquely mapped reads. MeDIP-seq data were analyzed using the R/Bioconductor package MEDIPS [25]. For each sample, the aligned reads were extended to a length of $300 \mathrm{bp}$ in the sequencing direction. The genome was divided into adjacent $500 \mathrm{bp}$ windows, and all additional calculations were applied to each window. Subsequently, methylation levels were quantified using MEDIPS to produce the relative methylation signal values (RMS) for further analysis. The mean relative methylation score (RPM) in each window across various regions of interest (e.g., promoters, 5'-UTR, 3'-UTR, exons, introns, CpG islands (CGIs)) was used to analyze the differentially methylated regions (DMRs).

\section{DMRs discovery and annotation}

For DMRs estimation, the RPM values in the control group ( $0 \mathrm{~d}, \mathrm{CK})$ were compared to each inoculated group stage I, II, and III. Differentially methylated regions were identified by applying edgeR for testing windows across regions of interest distributed throughout the genome. Significance of the results form DMRs analyses was estimated with $P$-value $<0.05$. Each DMR was annotated using Zea mays genes (AGPv3 (5b)) (http://plants.ensembl.org/ biomart/martview/242ebdeebecbf2ed59df0f7230470954).

\section{Digital gene expression (DGE) profiling data analysis}

DGE data were utilized from [22], and were reanalyzed in this study. Generally, the DGE data were processed using SOAP2 with default parameters. After removing adapters and low-quality reads, the clean tags were mapped to the maize reference genome v3 (RefGen_v3), then the uniquely 
mapped tags were normalized to TPM (number of transcripts per million clean tags), and used to analyze differentially expressed genes (DEGs) using edgeR [26]. The DEG results were estimated with a combination of FDR $<0.001$ and the absolute value of $\log 2-$ Ratio $\geq 1$. For further methylation analysis, all genes from DGE profiling mentioned below were differentially expressed genes. The analysis was followed that of Regulski et al. [27].

\section{Small RNA-seq data analysis and calculation of methylation in TEs}

The small RNA-seq data were utilized from [28] and reanalyzed in this study. Generally, data were filtered with SOAP2 using default parameters. The clean small RNA reads were mapped to the maize reference genome v3 (RefGen_v3) with a maximum of 2 mismatches. To estimate correlations between small RNA and methylation profiles in $2 \mathrm{~kb}$ upstream regions, the normalized read counts for small RNAs were used for calculations.

Reads mapped to transposable elements (TEs) were normalized as previously described [27].

\section{Data access}

The data from this study have been deposited in the NCBI Gene Expression Omnibus (GEO; http://www.ncbi.nlm.nih.gov/geo/) and are accessible through GEO Series accession number GSE84455.

\section{Results}

MeDIP-seq analysis of dedifferentiation in maize embryo reveals a large number of differentially methylated regions To investigate possible DNA methylation patterns changes that occur during callus induction in the maize embryo, we compared the methylated DNA of normal and inoculated embryos from the maize inbred line 18-599R using immunoprecipitation followed by massively parallel sequencing (MeDIP-seq). Samples were immediately collected $12 \mathrm{~d}$ after self-pollination, and inoculated embryos were collected at each stage (Fig. 1a; immature embryos without inoculation (CK), intumescent embryo (stage I), initial calli (stage II), and embryonic calli (stage III)) and were assessed with MeDIP-seq to generate a total of approximately $1.16 \times 10^{9}$ reads (average length $50 \mathrm{bp}$ ). The chromosomal distribution of DNA methylation reads for each maize embryo sample is depicted in Additional file 1: Fig. S1. In general, an average of $92.85 \%$ reads of the total reads aligned to the maize B73 reference genome, of which approximately $37.55 \%$ reads were uniquely mapped $\left(3.64 \times 10^{7}\right.$ reads; see Additional file 2: Table S1 for mapping statistics). To test for correlations between the MeDIP-seq samples, we calculated the Pearson's correlation coefficients based on read counts of the uniquely mapped reads. The results revealed a moderate to high overall similarity between samples $(r=0.56-0.92$; Additional file 2: Table S2). The pairwise correlations between MeDIP-samples derived from the same dedifferentiated stage were mostly above 0.80 . In contrast, the pairwise correlations between the CK group and each other stage were mostly below 0.80 , indicating a difference in global methylation after treatment.

To identify differentially methylated regions (DMRs) between the CK group and the other stages, we calculated and compared the read density in overlapping $500 \mathrm{bp}$ windows across the maize genome (described in the Methods section; $P<0.05$; mean signal in at least one group $>0.25$ reads per million; |ratio between CK and the other stage $\mid>2$ ). We identified 7036 differentially methylated regions (DMRs, size range $500 \mathrm{bp}$ ), of which 5376 (76.41\%) were hypermethylated and 1660 (23.59\%) were hypomethylated when comparing between stage I and the CK group (For example, see Fig. $1 \mathrm{~b}$ and $\mathrm{c}$ for a hyermethylated DMR in the promoter region of VIM1-like gene GRMZM2G461447). A total of 18,887 DMRs were identified in stage II (compared to CK), exhibiting 12,372 (65.51\%) hypermethylated and 6515 (34.49\%) hypomethylated regions; 11,514 DMRs were observed in stage III (compared to CK) with 9773 (84.88\%) hypermethylated and $1741(15.12 \%)$ hypomethylated regions (Table 1; see Additional file 2: Table S3 for full list of DMRs across different comparisons). Among these DMRs, 339 and 313 were consistently detected across all of the stages in the promoter region (Fig. 1d, upstream flanking 2000 bp region) and gene body regions (Fig. 1e), respectively. Moreover, 694,1121, 1371, and 1368 DMRs were uniquely present in promoter regions from CK, stage I, II, and III samples, respectively (Fig. 1d), whereas 1458, 3108, 2540, and 3483 DMRs uniquely appeared in the gene body regions from CK, stage I, II, and III embryos, respectively (Fig. 1e). Interestingly, we found 186 and 233 DMRs were consistent between all of the analyzed stages of callus induction in the promoter region and genebody region (Fig. 1d and e), respectively. Among these consistent DMRs, some may play important roles in the epigenetic manipulation due to the specificity to callus induction, such as dehydration-responsive element-binding protein 1B (DgDREB1B, GRMZM2G325513), which played an important role in plant development [29]; and 3methylcrotonyl-CoA carboxylase (MCCase, GRMZM2G 702490), a nuclear-encoded as well as mitochondrial biotin-containing enzyme, which has been reported the physiological roles in maintaining the carbon status of organism [30].

\section{Ontology-based enrichment analysis identified biological processes related to differential promoter methylation in embryonic callus formation}

The presence of DNA methylation is often considered to result in lower level of transcription. However, genomewide profiles of DNA methylation and gene expression 

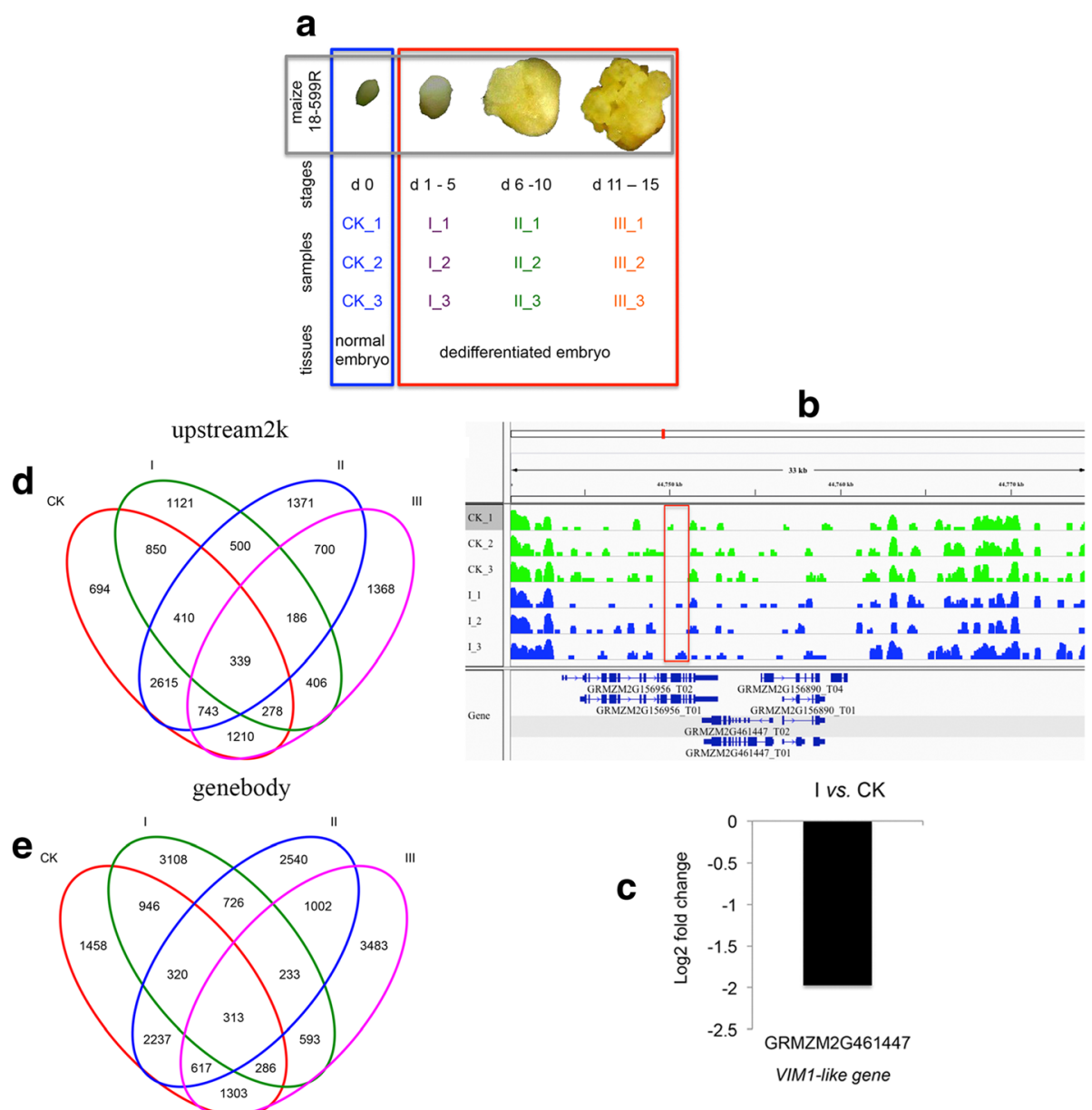

Fig. 1 Generation of genome-wide methylation maps for immature embryos and callui. a Summary of samples used for genome-wide methylation analyses. Normal embryo tissues (CK) and dedifferentiated embryo samples (I, II, and III) were employed for MeDIP-seq, DGE, and small RNA-seq. b Visualization of hypermethylated DMRs in the CK group and stage I-callus within the VIM1-like gene (GRMZM2G461447) using the IGV tool. Green, blue: MeDIP-seq tracks of the CK group and each embryo callus stage, respectively; red outlines the hypermethylated region of the gene of interest. c Expression of the VIM1-like gene, as determined by DGE. The expression is given as the log2-fold change calculated comparing the stage I-callus to the CK group (normal embryos). d-e Venn diagram showing the DMRs identified in $2 \mathrm{~kb}$ upstream and gene body regions

have suggested that DNA methylation does not cause decrease of gene expression during the functional stages $[10,15]$. Because we observed DMR enrichment in promoters (Additional file 2: Table S3), we performed gene ontology (GO) analysis on genes showing different promoter methylation using the Database for Annotation, Visualization and Integrated Discovery (DAVID) online tool (http://david.abcc.ncifcrf.gov/) to study the functional consequence of promoter methylation in an unbiased fashion [31]. Selected DAVID results are presented in Fig. 2, while all results are presented in Additional file 2: Table S4 $(P<0.05)$. Interestingly, for the hyper-methylated regions, the GO terms over-represented in comparisons Ivs.CKup and IIIvs.CKup analysis (e.g. cellular response to stress) seems more similar to each other than that in IIvs.CKup (e.g. regulation of transcription, DNA dependent). The function annotated from these comparisons by DAVID is consistent with the biological process of embryonic calli formation.

We found that the most enriched functional categories in the hypermethylation group were related to cellular responses to stress, DNA repair, DNA-dependent regulation of transcription, and responses to DNA damage, among others (Fig. 2a). Interestingly, we identified ion binding to be a uniquely enriched functional category in stage II (initial callus), which suggests that a number of genes, perhaps specifically encoding enzymes, might be involved in this process. Meanwhile, the finding of functions related to ARFs is interesting. In total, four genes (GRMZM2 G176495, GRMZM2G126079, GRMZM2G054821, GRMZ M2G083546) were observed to be enriched in the regulation of ARF protein signal transduction. We also performed $\mathrm{GO}$ analysis on the hypomethylation group and ranked the enriched GO terms according to their $p$-value (Fig. 2b). 
Table 1 Numbers of DMRs identified by MeDIP-seq, and assigned to subgenomic regions ${ }^{\mathrm{a}}$

\begin{tabular}{|c|c|c|c|c|c|c|c|}
\hline Comp. & DMR & Total & CGI & Promoter & Exon & Intron & $T T R$ \\
\hline \multirow[t]{3}{*}{ I vs. CK } & Total & 7036 & 830 & 2317 & 1684 & 1788 & 778 \\
\hline & Hyper & 5376 & 525 & 1830 & 1375 & 1254 & 664 \\
\hline & Нуро & 1660 & 305 & 487 & 309 & 534 & 114 \\
\hline \multirow[t]{3}{*}{ II vs. CK } & Total & 18,887 & 3293 & 6728 & 4440 & 4006 & 2040 \\
\hline & Hyper & 12,372 & 1213 & 4538 & 3315 & 2485 & 1696 \\
\hline & Нyро & 6515 & 2080 & 2190 & 1125 & 1521 & 344 \\
\hline \multirow[t]{3}{*}{ III vs. CK } & Total & 11,514 & 1419 & 3670 & 2899 & 2481 & 1376 \\
\hline & Hyper & 9773 & 1108 & 3142 & 2544 & 1963 & 1273 \\
\hline & Нyро & 1741 & 311 & 528 & 355 & 518 & 103 \\
\hline \multirow[t]{3}{*}{ |l vs. I } & Total & 5106 & 842 & 1413 & 1077 & 1440 & 432 \\
\hline & Hyper & 2234 & 143 & 731 & 568 & 628 & 237 \\
\hline & Нуро & 2872 & 699 & 682 & 509 & 812 & 195 \\
\hline \multirow[t]{3}{*}{ |II vs. II } & Total & 5765 & 1174 & 1643 & 1124 & 1595 & 453 \\
\hline & Hyper & 3667 & 1074 & 923 & 604 & 942 & 237 \\
\hline & Нyро & 2098 & 100 & 720 & 520 & 653 & 216 \\
\hline \multirow[t]{3}{*}{ III vs. I } & Total & 3742 & 363 & 969 & 874 & 1055 & 358 \\
\hline & Hyper & 2339 & 244 & 575 & 545 & 633 & 234 \\
\hline & Нуро & 1403 & 119 & 394 & 329 & 422 & 124 \\
\hline
\end{tabular}

${ }^{\mathrm{a} D M R}$, differentially methylated region; $\mathrm{CGl}, \mathrm{CG}$ island; $\mathrm{TTR}$, transcription termination region
The top enriched terms were relevant to RNA binding, phosphotransferase activity, and co-factor binding. This indicates that the phosphorylation of several factors including blue-light receptor phototropin 1 (phot1, GRMZM2G001457), blue-light receptor phototropin 2 (phot2, GRMZM2G032351), phytochromeC2 (phyC2, GR MZM2G129889), and histidine kinase1 (hk1, GRMZM2 G151223), is severely affected by tissue culture conditions. These results imply that factors responding stresses (e.g. darkness, auxin) and initiated in a DNA-methylation manner (e.g. through protein phosphorylation) might indirectly contribute to embryonic callus growth.

\section{Differential promoter methylation and differential gene} transcription in embryonic calluses are not highly correlated

It is generally assumed that promoter hypermethylation is correlated with down-regulation of the gene, whereas promoter hypomethylation is correlated with up-regulation $[14,17]$. However, this might not be true during maize embryonic callus development because a previous study provided little evidence to support consistent changes in maize DNA methylation patterns in response to performing different specific stress treatments [21]. To understand the effect of hypermethylation or hypomethylation on gene expression, we reanalyzed high throughput RNAsequencing data [22] on the same stages of tissue samples that were used for MeDIP-seq (see Additional file 1: Fig. S2

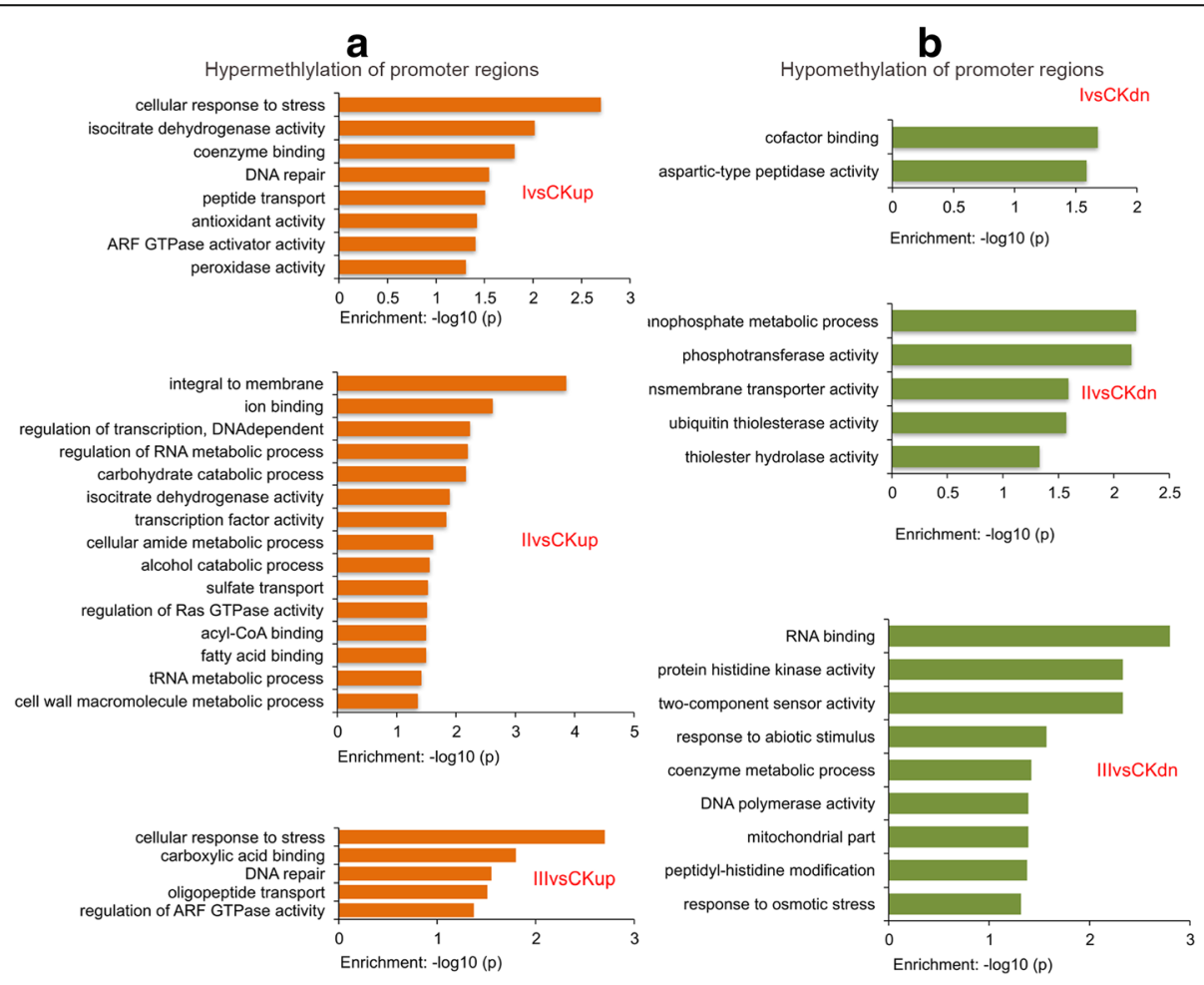

Fig. 2 Molecular features of genes with differentially methylated promoter regions in embryo calli. The genes with hypermethylated (a) or (b) hypomethylated promoter regions were analyzed by gene ontology, and the significantly enriched $(P<0.05)$ GO terms are plotted 
and Additional file 2: Table S5 for digital gene expression (DGE) data assessment and Additional file 2: Table S6 for list of differentially expressed genes). Generally, 1544 and 1523 genes were up-regulated (Additional file 1: Fig. S2A) and down-regulated (Additional file 1: Fig. S2B) in all stages of embryonic callus formation. The Kyoto Encyclopedia of Genes and Genomes (KEGG) pathway analyses resulted from DAVID online tool identified significantly overrepresented pathways relation to starch and sucrose metabolism, carbon fixation in photosynthetic organisms (Additional file 1: Fig. S2C) in the up-regulated genes and DNA replication, Citrate cycle (TCA cycle) in the downregulated genes (Additional file 1: Fig. S2D), respectively. We evaluated the genes that were, both differentially methylated and differentially expressed between the CK group and each embryonic callus stage (I, II, and III). The genes that were hypermethylated at their promoters and downregulated during callus induction has different numbers (Fig. 3a-c; 121 genes in stage I, 350 in stage II, and 246 in stage III). One example is the ZmEsr2 gene (CLAVATA3/ ESR (CLE)-related protein 2-B ESR2Bp, GRMZM2G 315601). Promoter hypermethylation is correlated with downregulation of the ZmEsr2 gene (Fig. 4), which is a known cytokinin-signaling molecule involved in developmental processes during maize embryo development [32, 33]. Likewise, promoter hypomethylation correlated with increased gene expression for several genes (15 genes in stage I, 123 in stage II, and 25 in stage III), but the overlap between genes with hypomethylated promoters and transcriptionally up-regulated genes was less extensive (Fig. 3a-c). However, some of the genes displayed a similar pattern between promoter hyper-methylation and down-transcriptional activity, or between hypomethylation and up-transcriptional activity, although some genes showed an inverse pattern. For example, $H 2 \mathrm{~A}$ (Histone H2A, GRMZM5G883764) contained a hypomethylated DMR in its promoter in stage II (compared to the CK group, Fig. 5a); however, this did not increase expression at stage II (Fig. 5b), although the gene plays an important role in dedifferentiated callus [34]. We also evaluated genes that were differentially expressed in the callus and that show changes to gene body methylation, although only a small overlap was observed between gene body methylation and gene expression (Fig. 3d-f).

\section{DNA hypermethylation in embryo calli occurs at genes} that might influence DNA methylation patterns in maize Previous data revealed that collections of mutant alleles for 11 maize genes were predicted to play roles in DNA methylation [35]. We thus assessed the promoter/gene body methylation and transcriptional activity of these genes potentially involved in maize DNA methylation. In the maize embryo-derived callus, however, none of these 11 genes were both promoter-hypermethylated and transcriptionally silenced, although the whole-genome methylation pattern showed greater hypermethylation in promoter regions compared to gene body regions (Fig. 6a). However, two out of the 11 genes (Chr106, GRMZM2G071025; Zmet5/ Dmt105, GRMZM2G005310) were both genebodyhypermethylated and transcriptionally down-regulated at stage II (Fig. 5c) and were not differentially expressed at other stages (compared to CK). Zmet5/Dmt105 is a fulllength chromomethylase gene in maize genome that is closely related to Arabidopsis CMT3, which is an important methytransferase [35]. Chr106 is similar to Arabidopsis $D D M 1$ and function as a chromatin remodeler.

Interestingly, the mediator of paramutation 3 (mop3, GRMZM2G007681) was hypermethylated in both the promoter and genebody regions during stage I (compared to CK) and hypermethylated in genebody regions in stage III (compared to CK); however, no mop3 transcriptional changes were observed during these two stages (Additional file 2: Table S3). Instead, the mop3 mRNA level was upregulated in stage II (compared to CK) (Additional file 2: Table S6), although we did not find any DMRs of this gene at stage II (compared to CK).

\section{Changes in DNA methylation levels at transposable elements differ after callus induction}

Transposable elements (TEs), which were first discovered in maize, are abundant and dynamic and play important roles in the evolution of genes and genomes in multiple organisms [36]. Previous studies found that methylation is guided by small RNAs and is correlated with transposon insertion [27]. We therefore asked whether the methylation signature of TEs were different; for instance, whether small RNAs guide methylation patterns during embryo callus formation (Fig. 6b). To this end, we identified that both type I and II TEs displayed hyper/hypo-methylation patterns during embryo callus formation. For type II transposons and type I transposons/SINE, extensive hypermethylation changes were observed at each embryo callus stage compared to CK, whereas hypermethylation of type I transposons/LINE only occurred at stage II (Fig. 6b). Type II TEs transpose by mobilizing DNA directly via a cut-andpaste mechanism, whereas type I TEs transpose by reverse transcription of a transcribed RNA [36, 37]. Other type I TEs,the major class of TEs called long terminal repeats (LTRs) retrotransposons [38], showed broad hypomethylation changes during each stage,with stronger hypomethylation at stage II (initial callus). Several studies demonstrated that type I elements, especially LTRs, contribute primarily to the dynamic gene function and evolution in higher plants. Some LTRs might amplify gene fragments and occasionally fuse to genes to create novel genetic functions [36, 39], leading to chromosomal rearrangements such as deletions, duplications, and translocations. Therefore, we further identified LTR subtypes as well as the other type I/ 


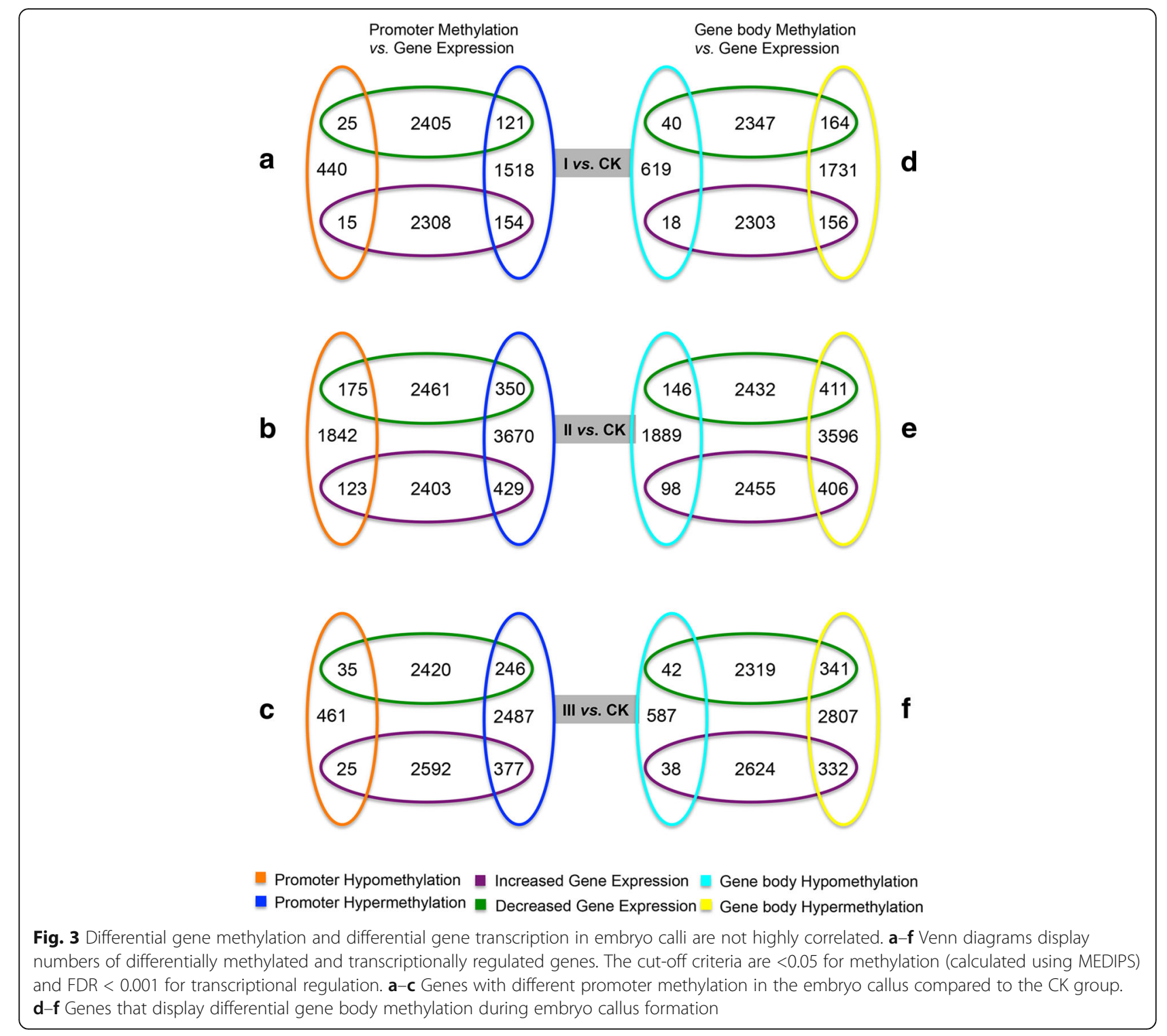

II TEs using the available maize transposable element database (http://maizetedb.org/ maize/) (Additional file 2: Table S7). Strikingly, we found that the majority of methylation level changes to TEs were at LTRs (see subtypes in Additional file 2: Table S7), suggesting potential roles for LTRs in embryonic callus formation.

Finally, we compared the levels of methylation with matching small RNAs [28] isolated from the same tissues as described in the Methods section. Small RNA data [28] generated from the same tissues used for MeDIP-seq (Additional file 2: Table S1) were mapped to the maize B73 genome (v3) and the transposable element database using Bowtie as previously described [27, 40]. Table 2 presents the correlations between 21, 22, 24-nt small RNAs and methylation. As shown in the table, the methylation level was not strongly correlated with 21-nt and 22-nt small
RNAs levels. However, similar to a previous study [27], 24nt small RNAs was significantly positively correlated with DNA methylation at each analyzed stage of callus induction but was negatively correlated with methylation in the CK group $(P<0.05$, Table 2$)$. To describe the targets of the 24nt small RNAs and to further describe the potential changes in expression in the pathways, we used a plant small RNA target analysis server (psRNATarget) [41] to map the target genes. All targets of the 24-nt small RNAs at each stage were listed in Additional file 2: Table S8. Finally, we identified 566 genes that are consistently targeted by 24-nt small RNAs among all the stages (stage I, II, and III, Additional file 1: Fig. S3). A previous study reported that the $24 \mathrm{nt}$ small RNAs are associated with RNAdependent DNA methylation (RdDM) that may give rise to transcriptional gene silencing. Furthermore, a study on the 
a
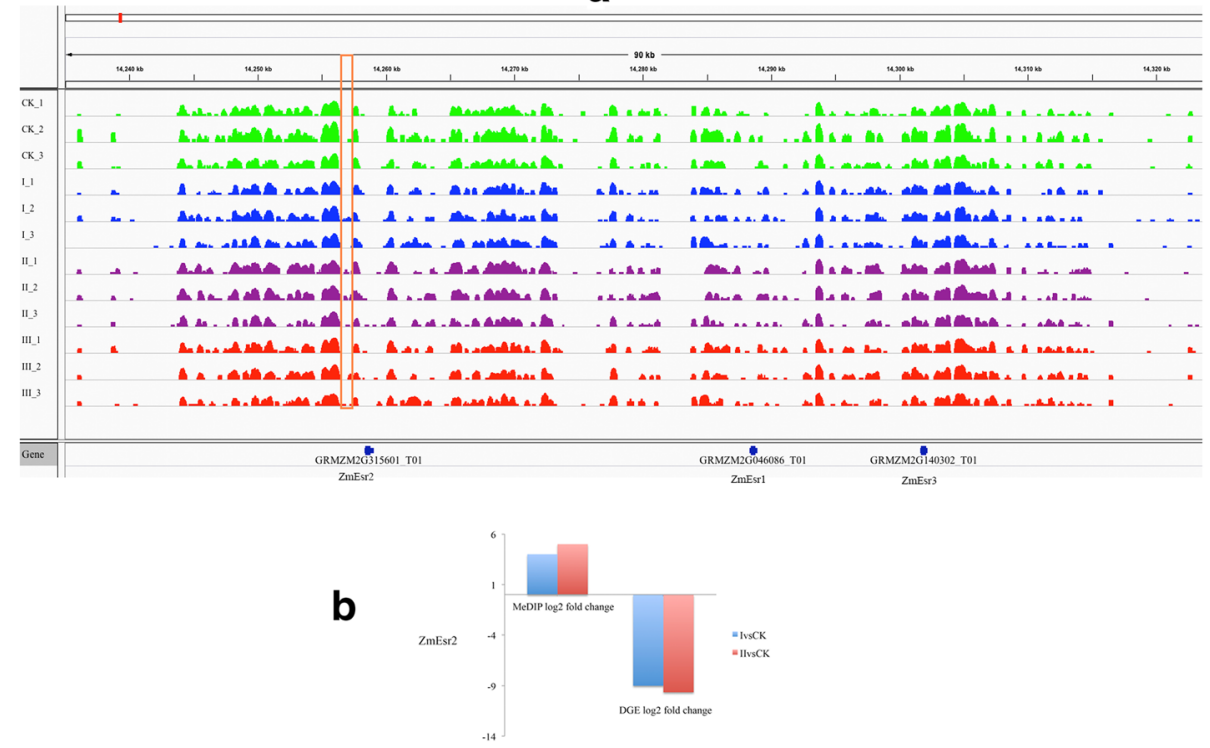

Fig. 4 Association of hypermethylation with transcriptional down-regulation at the ZmEsr2 locus. a Track of the MeDIP-seq data using the IGV tool. Green, blue,purple, and red: MeDIP-seq tracks for the CK group and each embryo callus stage; red outlines the hypermethylated region of the gene of interest. $\mathbf{b}$ Expression of ZmEsr2 and the neighboring ZmEsr1 and ZmEsr2 genes as determined by DGE. Expression is provided as the log2-fold change. ZmEsr2 was significantly down-regulated (FDR $<0.001)$

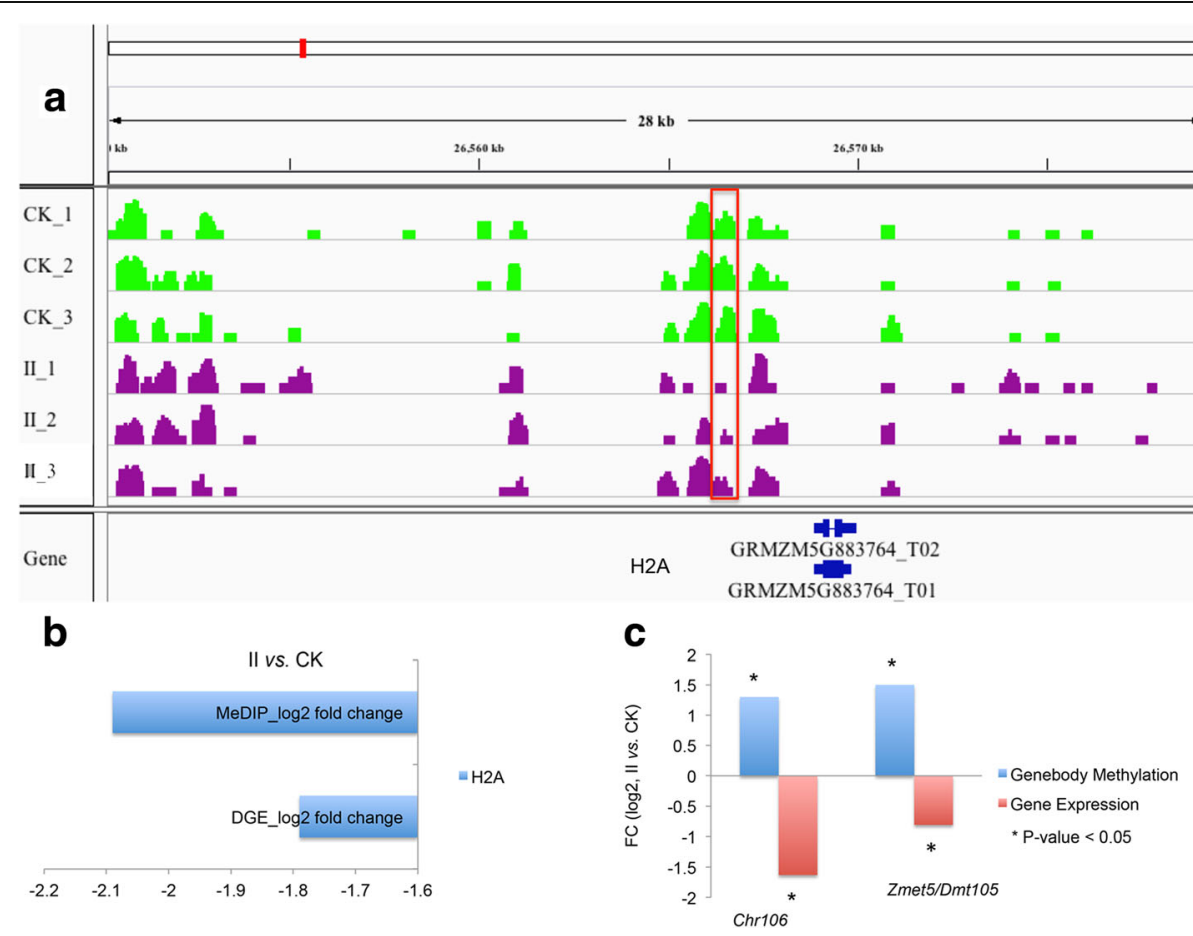

Fig. 5 Association of methylation and transcriptional down-regulation at a different locus. a IGV track of the MeDIP data at the H2A locus. Green, purple: MeDIP-seq tracks of the CK and stage II embryo callus groups, respectively; red outlines the hypomethylated region of the gene of interest. b H2A methylation and expression as determined by DGE. Expression is given as the log2-fold change as calculated for embryo callus compared to normal embryo. c Association of hypermethylation and down-regulation at the chr106 and Zmet5/ Dmt105 loci. Both genes are significantly down-regulated $(F D R<0.001)$ 

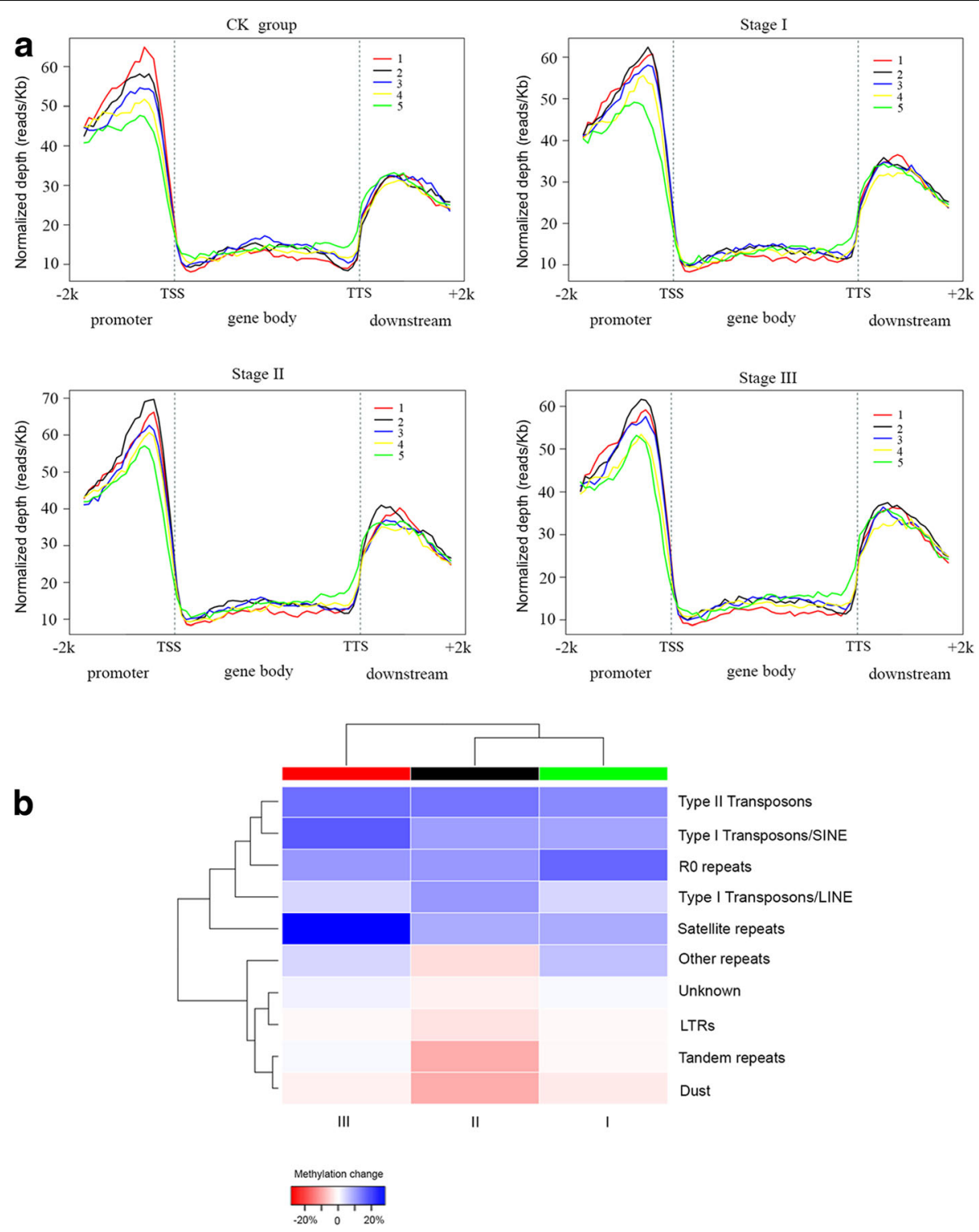

Fig. 6 Distribution of DNA methylation patterns in genes and TEs with different expression levels. a Gene expression levels (RPKM values) calculated from DGE data were classified into five categories, where "1" indicates the highest expression level and "5" indicates the lowest expression level. The $y$-axis represents normalized depth (reads/Kb). b Methylation change was calculated (as stage I, II or III - CK)/CK, and the values for each stage and the CK group are the average of the three replicates. The color red, black, green represent stage I, II, and III, respectively

Table 2 Small RNA guided methylation ${ }^{a}$

\begin{tabular}{|c|c|c|c|c|c|c|c|c|}
\hline \multirow{2}{*}{$\begin{array}{l}\text { Length of } \\
\text { small RNAs }\end{array}$} & \multicolumn{2}{|l|}{ CK } & \multicolumn{2}{|l|}{ I } & \multicolumn{2}{|l|}{$\|$} & \multicolumn{2}{|l|}{ III } \\
\hline & corrlation & $p$-value & corrlation & $p$-value & corrlation & $p$-value & corrlation & $p$-value \\
\hline 21 & 0.1404 & 0.5664 & 0.2407 & 0.3692 & 0.1815 & 0.4587 & -0.0194 & 0.941 \\
\hline 22 & -0.2584 & 0.2569 & 0.1956 & 0.3382 & 0.3652 & 0.0548 & 0.4434 & 0.016 \\
\hline 24 & -0.4285 & 0.0065 & 0.4821 & 0.0012 & 0.4522 & 0.0005 & 0.5309 & 0.0001 \\
\hline
\end{tabular}

${ }^{a}$ Correlation coefficents, calculated as described [26] 
root meristems of Arabidopsis thaliana indicated the significance of (24-nt) RNA silencing signal to embrace epigenetics and transcriptional gene silencing [42]. Intriguingly, pathway analysis of the identified 566 target genes results from DAVID indicates that the pathway zma03040: Spliceosome (http://www.genome.jp/kegg-bin/show_path way?map03040) was over-represented, which involved five target genes (GRMZM2G020728, GRMZM2G171372, GRMZM2G003307, GRMZM2G100620, GRMZM2G031 827). One of the players in the spliceosome pathway, splicing factor U2AF subunit (GRMZM2G031827), was found to be targeted by 24-nt small RNA (UAGGUUAUUCCUUUUGGUGUAGGC) and play a very important role in RNA splicing, indicates a potential novel signal where they caused epigenetic changes that may influence induction and development of maize embryo callus.

\section{Discussion}

For the first time, we compared methylated DNA from primary normal immature maize embryo to dedifferentiated cultures from the same organ using immunoprecipitation followed by massively parallel sequencing (MeDIP-seq). We observed that the callus-specific DNA methylation patterns were distinct from those found in normal immature embryos. These data indicate that callus-specific DMRs do not pre-exist in the cell population as a minor component of the maize embryo that emerge by expansion of the embryo callus cell type. These experiments establish that epigenetic patterns observed in dedifferentiated maize embryo cultures result from callus induction and will thus contribute to specific epigenetic manipulation.

Hypermethylation events were observed more frequently than hypomethylation events following callus initiation and formation during maize embryo dedifferentiation, which differs from embryonic callus formation for plant regeneration (re-differentiation process) but can ultimately be reflected in phenotypical variability of regenerated maize plants as described [43]. In our study, we mainly focused on the dedifferentiation process, which is characterized by more hypermethylation events. This might prepare the plant for later regeneration with increased hypomethylation, which is consistent with a previous study [43]. Stelpflug et al. [43] reported that decreased DNA methylation following tissue culture was more common than increase of DNA methylation during plant regeneration. For instance, indole-3-acetate beta-glucosyltransferase (GRMZM5G896260) was observed as hypermethylated DMR in the promoter region at stage III compared to the CK group, consistently, GRMZM5G896260 was detected as hypomethylated DMR (DMR ID 354) in the regenerated plant as described [43].

Generally, current epigenomic models assume that DNA hypermethylation, especially promoter methylation, is a negatively correlated with gene expression [17] and indicates gene silencing. We found that with respect to maize embryo calli, this promoter-model is only accurate for a minority of genes with hypermethylated promoters (Fig. 3a-c). Likewise, only a minor fraction of genes with hypomethylated promoters are transcriptionally up-regulated in embryo callus $($ Fig. $3 \mathrm{a}-\mathrm{c})$. These groups of genes occur more frequently in embryo calli than expected by chance; however, the large majority of detected genes do not follow conventional rules. Overall, changes in promoter methylation do not appear to significantly alter gene expression. Additional research is required to futher elucidate the regulation of gene expression by epigenetic mechanisms involving additional control elements such as enhancers and intragenic silencers in maize embryo calli.

Previous studies found that regions of DNA methylation within gene bodies were widely observed to have little to no influence on gene expression [15, 44, 45], whereas DNA methylation in the first hundred base pairs of a gene is associated with changes to gene expression [46]. Although the exact role of gene body methylation remains unclear, it might moderately influence transcribed genes $[14,17]$. However, we find the gene body model to be consistent with the rules as previously described $[14,17]$. A larger fraction of genes with genebody hypermethylation show changes in gene expression, whereas hypomethylation of the gene body leads to smaller changes in gene expression (Fig. 3d-f). This is an interesting phenomenon ignored by previous studies that should be thoroughly investigated in the future research on the maize epigenome, particularly in maize embryo dedifferentiation studies.

Although little to no correlation was observed between genebody methylation and gene expression, Regulski et al. [27] found that genebody methylation might prevent transposon insertion, disrupting gene function. Interestingly, Eichten et al. reported that genes located near retrotransposons were expressed at significantly lower levels in all of the examined maize genotypes and tissues [18], and DNA methylation differences associated with local genetic variation were observed near TEs [47]. In this study, we found substantial changes in methylation levels at transposable elements, most of which occurred at type I TEs/LTRs (Fig. 6) that are associated with chromosomal rearrangements such as deletions, duplications, and translocations [36, 39], which is consistent with previous reports [46].

\section{Conclusions}

In summary, our data define a core methylation signature of maize embryo dedifferentiation, which is of great importance for genetic manipulation. The comparison of immature embryo-derived callus with normal immature embryo indicated that this core signature is established early during embryonic callus formation and is retained when the embryonic callus epigenome is modified during embryo intumescence progression to embryonic callus. 


\section{Additional files}

Additional file 1: Fig. S1. Chromosomal distribution of DNA methylation read for each maize embryo sample. Each chromosomal was split in 10Kb windows. Fig. S2. Comparative and pathway analysis of DGE data. (A, B) Venn diagrams display the intersection of differentially expressed genes as determined by FDR $<0.001$ and log2fold change $>1$ for genes A) up-regulated and B) downregulated in differentiated embryo compared to normal embryo (CK group) (I vs. CK, II vs. CK, III vs. CK). C, D) KEGG pathway analyses. Overrepresented KEGG pathways in genes upregulated $(C)$ and down-regulated $(D)$ in differentiated embryos compared to CK group as calculated $(P<0.05)$ are shown. The $x$-axis displays the - $\log 10$ of the $p$-values calculated by DAVID (http://david.abcc.ncifcrf.gov). Fig. S3. Venndiagram of 24-nt small RNA target DMRs and pathway results from DAVID. Venn diagrams display the intersection of target genes of 24-nt small RNAs that significantly positive correlated with DMRs. (PDF $1136 \mathrm{~kb}$ )

Additional file 2: Table S1. Sequencing statistics of MeDIP-seq, mRNAseq, and small RNA-seq data. Tabel S2. Pairwise Pearson's correlation coefficients ( $r$ ) based on read counts of uniquely mapped reads. Table S3. Differentially methylated regions. Table S4. Data for Fig. 2. Table S5. Data for Additional file 1: Fig. S2. Table S6. DGE in stages vs. CK. Table S7. TE changes in stages vs.CK. Table S8. Data for Additional file 1: Fig. S3. (XLS 15783 kb)

\section{Abbreviations}

CGI: CpG island; CK: Control group; DAVID: Database for Annotation, Visualization and Integrated Discovery; DEG: Differentially expressed genes; DGE: Digital gene expression; DMRs: Differentially methylated regions; GO: Gene ontology; KEGG: Kyoto Encyclopedia of Genes and Genomes; LTRs: Long terminal repeats; MeDIP-seq: Methylated DNA immunoprecipitation sequencing; RdDM: RNA-dependent DNA methylation; RMS: Relative methylation signal values; RPM: Mean relative methylation score; TE: Transposable element; TPM: Number of transcripts per million clean tags; TTR: Transcription termination region

\section{Acknowledgements}

The authors thank Drs. Tao Zuo and Qing Li for critical reading of manuscript. We thank Huangkai Zhou for help with the data analysis and Qiong Wang for help with the plant material. We also thank Shanghai Novelbio Ltd. for help with the data interpretation.

\section{Funding}

This work is supported by the National Natural Science Foundation of China (31471512), the Young Scientists Fund of Sichuan Province (2016JQ0008), the Major Project of China on New varieties of GMO Cultivation (2016ZX08003003), the Talent Project Funded by Shandong Agricultural University (72127), and the Funds of Shandong 'Double Tops' Program.

\section{Availability of data and materials}

The data from this study have been deposited in the NCBI Gene Expression Omnibus (GEO; http://www.ncbi.nlm.nih.gov/geo/) and are accessible through GEO Series accession number GSE84455.

\section{Authors' contributions}

YS, GP, and HLiu designed the study. HLiu, LM, XY, LZ, XZ, SX, and YW performed the analyses. HLiu, XY, HP, and YS drafted the manuscript. SG, HLin, and SX help to interpret the data. All authors critically revised and provided final approval of this manuscript.

\section{Competing interests}

The authors declare that they have no competing interests.

\section{Consent for publication}

Not applicable.

Ethics approval and consent to participate Not applicable.

\section{Publisher's Note}

Springer Nature remains neutral with regard to jurisdictional claims in published maps and institutional affiliations.

\section{Author details}

${ }^{1}$ Key Laboratory of Biology and Genetic Improvement of Maize in Southwest Region, Maize Research Institute, Sichuan Agricultural University, Chengdu 611130, China. ${ }^{2}$ State Key Laboratory of Crop Biology, College of Life Sciences, Shandong Agricultural University, Tai'an 271018, China.

${ }^{3}$ Department of Agronomy, Northeast Agricultural University, Harbin 150030, China. ${ }^{4}$ Suihua Sub-academy, Heilongjiang Academy of Agricultural Sciences, Suihua 152052, China. ${ }^{5}$ Institute of Animal Nutrition, Sichuan Agricultural University, Ya'an 625014, China. ${ }^{6}$ National Key Laboratory of Plant Molecular Genetics, Institute of Plant Physiology \& Ecology, Shanghai Institutes for Biological Sciences, Chinese Academy of Sciences, Shanghai 200032, China.

Received: 29 January 2017 Accepted: 6 June 2017

Published online: 15 June 2017

\section{References}

1. Ahmadabadi M, Ruf S, Bock R. A leaf-based regeneration and transformation system for maize (Zea mays L.). Transgenic Res. 2007:16(4):437-48.

2. Buckler ES, Gaut BS, McMullen MD. Molecular and functional diversity of maize. Curr Opin Plant Biol. 2006;9(2):172-6.

3. Messing J, Dooner HK. Organization and variability of the maize genome. Curr Opin Plant Biol. 2006;9(2):157-63.

4. Rabinowicz PD, Bennetzen JL. The maize genome as a model for efficient sequence analysis of large plant genomes. Curr Opin Plant Biol. 2006:9(2):149-56.

5. Schnable PS, Ware D, Fulton RS, Stein JC, Wei F, Pasternak S, et al. The B73 maize genome: complexity, diversity, and dynamics. Science. 2009; 326(5956):1112-5.

6. Kaeppler SM, Phillips RL. Tissue culture-induced DNA methylation variation in maize. Proc Natl Acad Sci U S A. 1993:90(19):8773-6.

7. Richards EJ. Inherited epigenetic variation-revisiting soft inheritance. Nat Rev Genet. 2006;7(5):395-401

8. Feinberg AP. Phenotypic plasticity and the epigenetics of human disease. Nature. 2007:447(7143):433-40.

9. Jirtle RL, Skinner MK. Environmental epigenomics and disease susceptibility. Nat Rev Genet. 2007:8(4):253-62.

10. Vaughn MW, Tanurdzic M, Lippman Z, Jiang H, Carrasquillo R, Rabinowicz PD, et al. Epigenetic natural variation in Arabidopsis thaliana. PLoS Biol. 2007:5(7):e174.

11. Johannes F, Colot V, Jansen RC. Epigenome dynamics: a quantitative genetics perspective. Nat Rev Genet. 2008:9(11):883-90.

12. Stroud H, Greenberg MV, Feng S, Bernatavichute YV, Jacobsen SE. Comprehensive analysis of silencing mutants reveals complex regulation of the Arabidopsis methylome. Cell. 2013;152(1-2):352-64.

13. Lippman Z, Gendrel AV, Black M, Vaughn MW, Dedhia N, McCombie WR, et al. Role of transposable elements in heterochromatin and epigenetic control. Nature. 2004;430(6998):471-6.

14. Zhang X, Yazaki J, Sundaresan A, Cokus S, Chan SW, Chen H, et al. Genomewide high-resolution mapping and functional analysis of DNA methylation in arabidopsis. Cell. 2006;126(6):1189-201.

15. Zilberman D, Gehring M, Tran RK, Ballinger T, Henikoff S. Genome-wide analysis of Arabidopsis thaliana DNA methylation uncovers an interdependence between methylation and transcription. Nat Genet. 2007;39(1):61-9.

16. Eichten SR, Foerster JM, de Leon N, Kai Y, Yeh CT, Liu S, et al. B73-Mo17 near-isogenic lines demonstrate dispersed structural variation in maize. Plant Physiol. 2011;156(4):1679-90.

17. Eichten SR, Swanson-Wagner RA, Schnable JC, Waters AJ, Hermanson PJ, Liu $\mathrm{S}$, et al. Heritable epigenetic variation among maize inbreds. PLoS Genet. 2011;7(11):e1002372

18. Eichten SR, Ellis NA, Makarevitch I, Yeh CT, Gent JI, Guo L, et al. Spreading of heterochromatin is limited to specific families of maize retrotransposons. PLoS Genet. 2012;8(12):e1003127.

19. Eichten SR, Vaughn MW, Hermanson PJ, Springer NM: Variation in DNA methylation patterns is more common among maize inbreds than among tissues. The plant genome 2013, 6(2).

20. Li Q, Eichten SR, Hermanson PJ, Springer NM. Inheritance patterns and stability of DNA methylation variation in maize near-isogenic lines. Genetics. 2014;196(3):667-76. 
21. Eichten SR, Springer NM. Minimal evidence for consistent changes in maize DNA methylation patterns following environmental stress. Front Plant Sci. 2015;6:308.

22. Shen $Y$, Jiang Z, Yao X, Zhang Z, Lin H, Zhao M, et al. Genome expression profile analysis of the immature maize embryo during dedifferentiation. PLoS One. 2012;7(3):e32237.

23. Li N, Ye M, Li Y, Yan Z, Butcher LM, Sun J, et al. Whole genome DNA methylation analysis based on high throughput sequencing technology. Methods. 2010;52(3):203-12.

24. Li R, Yu C, Li Y, Lam TW, Yiu SM, Kristiansen K, et al. SOAP2: an improved ultrafast tool for short read alignment. Bioinformatics. 2009;25(15):1966-7.

25. Lienhard M, Grimm C, Morkel M, Herwig R, Chavez L. MEDIPS: genome-wide differential coverage analysis of sequencing data derived from DNA enrichment experiments. Bioinformatics. 2014;30(2):284-6.

26. Robinson MD, McCarthy DJ, Smyth GK. edgeR: a Bioconductor package for differential expression analysis of digital gene expression data. Bioinformatics. 2010;26(1):139-40.

27. Regulski M, Lu Z, Kendall J, Donoghue MT, Reinders J, Llaca V, et al. The maize methylome influences mRNA splice sites and reveals widespread paramutationlike switches guided by small RNA. Genome Res. 2013;23(10):1651-62.

28. Shen $Y$, Jiang Z, Lu S, Lin H, Gao S, Peng H, et al. Combined small RNA and degradome sequencing reveals microRNA regulation during immature maize embryo dedifferentiation. Biochem Biophys Res Commun. 2013; 441(2):425-30.

29. Tong Z, Hong B, Yang Y, Li Q, Ma N, Ma C, et al. Overexpression of two chrysanthemum DgDREB1 group genes causing delayed flowering or dwarfism in Arabidopsis. Plant Mol Biol. 2009;71(1-2):115-29.

30. Che P, Wurtele ES, Nikolau BJ. Metabolic and environmental regulation of 3methylcrotonyl-coenzyme a carboxylase expression in Arabidopsis. Plant Physiol. 2002;129(2):625-37.

31. Huang da W, Sherman BT, Lempicki RA. Systematic and integrative analysis of large gene lists using DAVID bioinformatics resources. Nat Protoc. 2009; 4(1):44-57.

32. Opsahl-Ferstad HG, Le Deunff E, Dumas C, Rogowsky PM. ZmEsr, a novel endosperm-specific gene expressed in a restricted region around the maize embryo. The Plant journal : for cell and molecular biology. 1997;12(1):235-46.

33. Wang G, Fiers M. CLE peptide signaling during plant development. Protoplasma. 2010;240(1-4):33-43.

34. Alatzas A, Foundouli A. Distribution of ubiquitinated histone $\mathrm{H} 2 \mathrm{~A}$ during plant cell differentiation in maize root and dedifferentiation in callus culture. Plant science : an international journal of experimental plant biology. 2006; 171(4):481-7.

35. Li Q, Eichten SR, Hermanson PJ, Zaunbrecher VM, Song J, Wendt J, et al. Genetic perturbation of the maize methylome. Plant Cell. 2014;26(12):4602-16.

36. Baucom RS, Estill JC, Chaparro C, Upshaw N, Jogi A, Deragon JM, et al. Exceptional diversity, non-random distribution, and rapid evolution of retroelements in the B73 maize genome. PLoS Genet. 2009;5(11):e1000732.

37. Kapitonov W, Jurka J. Rolling-circle transposons in eukaryotes. Proc Natl Acad Sci U S A. 2001;98(15):8714-9.

38. Bennetzen JL. Transposable elements, gene creation and genome rearrangement in flowering plants. Curr Opin Genet Dev. 2005;15(6):621-7.

39. Wang W, Zheng H, Fan C, Li J, Shi J, Cai Z, et al. High rate of chimeric gene origination by retroposition in plant genomes. Plant Cell. 2006;18(8):1791-802.

40. Langmead B, Trapnell C, Pop M, Salzberg SL. Ultrafast and memory-efficient alignment of short DNA sequences to the human genome. Genome Biol. 2009;10(3):R25.

41. Dai $X$, Zhao PX: psRNATarget: a plant small RNA target analysis server. Nucleic acids research 2011, 39(Web Server issue):W155-159.

42. Melnyk CW, Molnar A, Bassett A, Baulcombe DC. Mobile 24 nt small RNAs direct transcriptional gene silencing in the root meristems of Arabidopsis thaliana. Current biology : CB. 2011;21(19):1678-83.

43. Stelpflug SC, Eichten SR, Hermanson PJ, Springer NM, Kaeppler SM: Consistent and Heritable Alterations of DNA Methylation Are Induced by Tissue Culture in Maize. Genetics. 2014.

44. Hollister JD, Smith LM, Guo YL, Ott F, Weigel D, Gaut BS. Transposable elements and small RNAs contribute to gene expression divergence between Arabidopsis thaliana and Arabidopsis lyrata. Proc Natl Acad Sci U S A. 2011;108(6):2322-7.

45. Yang L, Takuno S, Waters ER, Gaut BS. Lowly expressed genes in Arabidopsis thaliana bear the signature of possible pseudogenization by promoter degradation. Mol Biol Evol. 2011;28(3):1193-203.
46. Li Q, Song J, West PT, Zynda G, Eichten SR, Vaughn MW, Springer NM: Examining the causes and consequences of context-specific differential DNA methylation in maize. Plant physiology 2015.

47. Eichten SR, Briskine R, Song J, Li Q, Swanson-Wagner R, Hermanson PJ, et al. Epigenetic and genetic influences on DNA methylation variation in maize populations. Plant Cell. 2013;25(8):2783-97.

\section{Submit your next manuscript to BioMed Central and we will help you at every step:}

- We accept pre-submission inquiries

- Our selector tool helps you to find the most relevant journal

- We provide round the clock customer support

- Convenient online submission

- Thorough peer review

- Inclusion in PubMed and all major indexing services

- Maximum visibility for your research

Submit your manuscript at www.biomedcentral.com/submit
Biomed Central 